\title{
Tricky Sexual Differences: What Is Perversion?
}

The purpose of this paper is to explicate in detail why we should not use - or even mention - the terms perversion and pervert: these are not only essentially contested but inherently discriminatory terms. Thomas Nagel's famous article "Sexual Perversion" is an often reprinted contribution to the philosophy of sexuality. I critically review his arguments and compare them with those of Sigmund Freud and others. He explains the genesis of perversions based on developmental trauma. Freud does not identify and label perverted persons in such a simplicistic manner as he admits that healthy persons may play with perverted desires. Yet, he also finds perversions pathological. All this talk should change because hetero vanilla sex cannot be the only viable type of sex. Of course, evil sexual practices exist, like rape, but this has nothing to do with perversity. Also sex and love are two separate things.

\section{The Tropes of Perversity}

Sexuality is a touchy cultural topic loaded with a moralistic package, and therefore also strictly regimented by the church (mortal sin), positive law (crime), natural law (unnatural behaviour), tradition and its mores (dirty and disgusting practices ${ }^{1}$ ). Medical lore follows suit: certain sexual desires are called paraphilias or paraphilic disorders as if they were pathologically symptomatic thus deserving a fancy name. ${ }^{2}$ For instance, BDsm (Bondage, Discipline, Sadism, and Masochism, also D/S or contractual domination and submissiveness) has been classified together with fetishism, paedophilia, zoophilia, coprophilia or scatology etc., so offensive is its popular image. For instance, the scatological fetish, or playing with human excrement, may be difficult to accept as normal. ${ }^{3}$

1 Disgust is the key term when we discuss perversion, see for instance R. Herz, That's Disgusting. New York: Norton, 2012, Ch. 6 "Lust and Disgust." Dirt stains and pollutes a person and brands him more or less permanently. Labels like "pervert" stick.

2 For the state of the medical debate on this, see S. Wright, "Depathologizing Consensual Sexual Sadism, Sexual Masochism, Transvestic Fetishism, and Fetishism," Arch Sex Behav (2010) 39:1229-1230; and U. Khan, "Sadomasochism in Sickness and in Health: Competing Claims from Science, Social Science, and Culture," Curr Sex Health Rep (2015) 7:49-58.

3 Scatology is an essential if nauseating part of M. de Sade's idea of "simple pleasure"; see the early days and weeks of the 120 Days of Sodom. See also S. Freud, On Sexuality, Three Essays on 
Is BDSM a perversion? Is it a paraphilia? Is it a paraphilic disorder? The best answer may well be in the negative; it is kinky and perhaps weird but these two terms are not necessarily condemnatory; their tropology today may be ironic, intentionally subversive, and multi-dimensionally suggestive, they may be socially unmanageable and even creatively playful. We may also call BDSM minority sex because minority is a relational or non-essential term - the situation may change when social conventions and customs change, like in the case of gay sex in many liberal cultures of today. However, calling BDSM perverse or paraphilic sex and a paraphilic disorder assigns it a non-relational or essentially negative, unique and ultimately discriminatory characterization. An argument against such a negative rhetorical strategy exists: it is ad hoc. If you want to censure or condemn a type of wish, desire, need, or action you should not use a term that is tailor-made for this special purpose only; it is called negative labelling, which as such is an ad hoc strategy devoid of explanatory weight. The strategy has Biblical roots: to call a desire a sin is to label it as something against the religious canon; the next step is to metonymically relate sin to immorality; and thus a sectarian condemnation turns into a universalizable moral judgement. In this way, sin becomes applicable to everyday social life achieving in the process an extra-religious moralistic meaning tone that also seems to justify its use. Sin extends its halo into the fields of morality and the law. This happens to perversion and, to a lesser degree, to paraphilia as well.

It is customary to describe a kinky sexual activity in common negative terms, which make its disapproval appear not prejudiced but objective and coherent. However, one must avoid labelling or applying special categorical terms in an ad hoc manner, terms that work in a given context only. For instance, by calling BDSM sick, unnatural, immoral, or sinful you make it one of the activities condemned by the medical community, concerned citizens, and some Christian churches. ${ }^{4}$ Call it ugly, disgusting, criminal, and insane and you attract a different audience. You should specify the reasons for your condemnation because no bad thing can be bad in its own unique way and in its own special mode, like the perverse or paraphilic activities allegedly are. But such specification may not be easy if you do not lean heavily on negative emotional terms.

When one uses the term perverse, one uses a term that condemns certain desires and actions within the broad and wide open field of sexuality and eroticism. Such a usage is, tropologically speaking, fetishist. The word works as a

the Theory of Sexuality, Pelican Freud Library Vol. 7. Tr. J. Strachey. London: Penguin Books, 1977, p. 104, and about "disappearance of their anal eroticism," in the case of those neurotics who are now "orderly, parsimonious, and obstinate" (p. 210).

4 See E. Berkowitz, Sex and Punishment. London: Westbourne Press, 2013. 
linguistic and rhetorical talisman, a magic name that is required to do one special job only: to place a negative and discriminatory label on something that is already abhorred, or required to be so. This is because perversion has no independent meaning base. As a verbal talisman and fetish it does not; instead, we create a new use for the word, or a new word, that has no independent meaning base, no previous use or history. This is what a verbal fetish does, it works as if in a vacuum where its emerging effect looks like a kind of miracle. In the end, the irony of this all is that BDSM appears as a type of sexual fetishism, which condemns it as a perversion that in itself is a fetishist trope. Hence, to speak of perversions is perverted, which is nothing but criticism's suicide.

Perversion talk as a linguistic performative convinces and condemns without a reason or ground. Perhaps the general non-sexual meaning of perversion, if it has a discursive meaning, is something like happily but incomprehensibly violating one's own self or essential interests? A meaning like that can be coined but a glance at a thesaurus shows that perversion fails to have proper synonyms; the best I can find is distortion. BDSM would then be distorted sex, but along with such a trope we certainly approach the fuzzy realms of metaphor. If perversion means a hopelessly twisted affair it does not say much about sex; sex tends to be hopelessly twisted anyway in all its forms. That is why we talk about vanilla sex, but who says vanilla sex is a simple thing? ${ }^{5}$

I suppose the terms paraphilia and paraphilic disorder fare a little better in this respect but then they are technical medical terms that should be carefully unpacked in order to see what they cover and hide. Why coin such terms? Why not use statistical terms such as rare and uncommon? Even strange, non-standard, and abnormal might do. The question is, even if we prioritize vanilla sex, or sex that is or simulates human reproductive activity, what is the reason for condemning all the other sources of sexual pleasure? Why provide them with a common characterization after all? No good answer exists. It has been suggested, of course, that paraphilic disorders are personally painful and harmful in the long run, and thus the sufferer tends to seek for help. They are in this sense like diseases. This may well be so but different people react differently to different activities. Some individuals flourish, some suffer, so that it is difficult to say anything informative about an activity like BDSM. A happy voyeur may

5 A paradox of perversion: if perversion is sick sex, or deviant sex, one may desire it exactly because of this - the more sick and deviant the better. Perversion as the new normal would deprive perverts of their desire and pleasure; see J. Dollimore, Sex, Literature and Censorship. Cambridge: Polity Press, 2001, pp. 8 fff. The problem is that this applies to vanilla sex as well. For some people all non-procreative sex is exciting and normatively ambiguous, think of devout Catholics. The other side of the paradox is that deviant desires may be a source of great pain. Can one want sick sex because of its painfulness? 
exist along with others who suffer because of their desire. If they cause true social harm, they commit a misdemeanour and crime, which is an altogether different worry. The best rule of thumb is, as it seems to me, if BDSM related desire brings about intolerable pain, suffering, and harm (de se) to a person that calls for psychotherapy and medical help. However, even in this case one might want to resist the temptation of calling BDSM the problem. The relevant, in this case harmful, activities need not be condemned in a broader context. The sexual disorder is this person's own personal problem. In fact, it is his own problem like any other problems he may have. We may call it a BDSM generated problem; say, deep anxiety and depression, just like we call a broken leg a fall related bone problem. Yet, one cannot say BDSM is the problem because happy BDSM aficionados exist, we know that. BDSM is not like a broken leg.

\section{Freud: Perverted and Pathological Desires}

Any account of any type of perversion provided by the members of its nonintended audience tends to be philosophically and analytically objectionable. The same can be said of scholarly definitions. I give two examples, a classic psychoanalytical definition by Sigmund Freud, and later on the famous philosophical one by Thomas Nagel. Other definitions exist but these two are, as far as I can see, representative and certainly influential. Sigmund Freud first notices that obviously healthy people may make certain strange additions to their otherwise normal and healthy vanilla sex centred sexual repertoire. ${ }^{6}$ Unlike Nagel, he maintains that they are normal, except that their sexual life is or tends to be abnormal, and, hence, sexual perversions do not always require a psycho-pathological aetiology or signify anything like mental illness. Hence, perverted sex is predicated on desires, activities, and practices, and not on persons. Nevertheless, Freud says that many sexual desires and practices are "so far removed from the normal in their content that we cannot avoid pronouncing them 'pathological:" ${ }^{7}$ Here a dubious emphasis is placed on non-statistical and pseudo-normative reference to something called normal. This is the central trope in conservative contexts whose key purpose is to put a lid on the life and language of some activities that are too shameful to mention. Again, Freud

6 He repeats this idea, see Freud, 1977, p, 74, also pp. 61-62, 66, 74, 87. Ultimately, it sounds as if perversion is internal to all sexuality, which comes close to saying that no healthy or normal sexuality exists.

7 Freud, 1977, p. 74. This is a bad idea, for obvious reasons. A gloriously beautiful person is not pathological. A mathematical genius may not be pathological. 
says that some practices, and not people, are sick and so, metonymically, they make people sick. Such desires and practices should carry a label "Do Not Try!" Fetishism, including some aspects of BDSM, certainly belongs to this category almost by definition: a fetishist attaches sexual value to non-sexual things and actions, which, consequently, are "so far removed from the normal." The idea is clear: A woman's shoe - an industrial artefact - is indeed far removed from normal sexual objects.

Freud's definition of perversion, or abnormal and pathological sex, contains a preliminary point and two main parts. First, perversion metonymically replaces vanilla sex taking its role in life and language. Then the first part of the definition itself: perversion entails a "sexual instinct [...] overriding the resistance or shame, disgust, horror, and pain"; and the second part: "perversion has the characteristic of exclusiveness and fixation." Especially the second part should justify the epithet perverse as a pathological symptom. ${ }^{8}$ But a person may show, in vanilla sex, a strong fixation to the partner's genitals - obviously Freud does not mean this. Perversions are supposed to be endlessly repetitive and fully rigid and in this sense demeaning, inhuman, or machine like - when machine becomes the hidden key metaphor in this context. Only machines are fully rigid. This is interesting even if it does not really apply to BDSM, as it easy to verify. The members of BDSM community can be and are fully flexible with their desires, say, a top and a bottom may change their roles (see Ch. 10). ${ }^{9}$ Nevertheless, active BDSM community members are able to deal with "shame, disgust, horror, and pain" - this is the name of their game. A submissive person is supposed to enjoy disgusting humiliation. Therefore, what Freud is afraid of they desire, which is a source of irony here. In fact, Freud emphasizes disgust as an essential component in connection with perversions. ${ }^{10}$

Notice that shame and disgust apply to many or most people who are starting their sex lives; it can be hard to perform a complete fellatio (give head) or cunnilingus (eat pussy) and enjoy it. In vanilla sex, genitals may remain a source of disgust, which the sexual lust then overrides. But you do not become perverted when you get used to it. Freud's, and we will see, Nagel's definitions are conservative and perhaps logically dubious. Their main purpose is to justify the desire for vanilla sex by making its alternatives look bad to the nonintended audiences of the type of sex play in question.

$8 \quad$ S. Freud, 1977 , p. 75 ; also pp. 66-67 and p. 75 .

9 Freud (1977, p. 73) says that a "sadist is always at the same time a masochist," and that entails flexibility.

$10 \quad$ Freud, 1977, pp. 64, 76 etc. 


\section{Nagel: the Substantiality Thesis}

Thomas Nagel's "Sexual Perversion" (SP in what follows) has been reprinted numerous times and widely quoted. ${ }^{11} \mathrm{He}$ has a mission, namely, to reveal the secrets of good sex and not only good sex but ethically sustainable sex as well. The paper seems to have a special appeal to a wide range of specialists in the philosophy of sex and sexuality. Therefore, I ask a simple question, namely, is this justified? If it is, we should find a solid and valid analysis of good sex and a convincing reason to reject a wide variety of sexual desires and practices because they are perverted. Concerning sexual perversions in SP, I find three layers: first, Nagel defends the idea that the idea of sexual perversion is a substantial one; that is, it is not a mere colloquial pejorative term. Second, he provides us a narrative account of non-perverted and ideal sexual behaviour. Third, he tells us what sexual perversion is and what causes it. I deal with these points one by one.

Let us now look into the details of SP. The first argument has two parts, first the argument from anti-relativity and then the argument from generalization. Nagel writes,

What is regarded as unnatural admittedly varies from culture to culture, but the classification is not a pure expression of disapproval of distaste. In fact it is often regarded as a ground for disapproval, and that suggests that the classification has independent content. ${ }^{12}$

The key concept here and throughout the paper is unnatural. Anyway, the first part of Nagel's substantiality argument says that however much different cultures and times vary in terms of their sexual ethics they all use a notion of unnatural sex, which they disapprove. They have their ideas about good and acceptable sex as well as on bad and forbidden sex, or unnatural, sex. We have found a cultural universal. Often they reject something because of the violation of a taboo, for instance. Unnatural as a condemnatory term may not have such a universal appeal as Nagel believes. ${ }^{13}$ Roman Catholic philosophers use the term freely but that is another thing. Be this as it may, the main point is that

11 T. Nagel, "Sexual Perversion" (in his Mortal Questions. Cambridge: Cambridge University Press, 1979) has been recently reprinted, for instance, in such a major textbook as G. Foster, Ed., Desire, Love, and Identity. New York: Oxford University Press, 2016.

12 Nagel, 1979, p. 40.

13 Well, this is easy to say, but look for instance M. Douglas, Purity and Danger. London: Routledge, 1966/2008, p. 175: "The people [Walbiri of Central Australia] have no beliefs concerning sexual pollution." 
in many cultures the tit-for-tat arousal between man and woman is considered weird or even impossible. Ancient Greeks may have thought so. Plato hated painted ladies and desired beautiful young men even if he hardly imagined that they would desire him. Are these people perverted?

The second part of the argument for substantiality is the generalization thesis, which begins like this: "Can we imagine anything that would qualify as gastronomical perversion?"14 Nagel answers his own question thus: "Even an appetite can have perversions if in addition to its biological function it has a significant psychological structure." I am not sure this is true of appetite for food. We do not use the term perversion in any serious sense in connection with food. Sex is, indeed, a special case. I am not a pervert if I desire dog meat, bull's balls, or pig's trotters. If one eats excrement one is probably psychotic. If one entertains sexual interests in a dog or pig, one is, colloquially speaking, a pervert. Perversion indeed is, when it us used seriously, a special negative term dedicated to sexuality. Nagel has not shown the substantial and generalized nature of the concept of perversion.

The gastronomical part of SP is important because it hints at what Nagel really is after, and that is his ideal sex as love. Culturally I see it as a typical Hollywood Americanism. Sex without love is dirty business. Perhaps this has something to do with the fact that that porn industry flourishes in the US but prostitution is strictly illegal, except in the great state of Nevada. Anyway, Nagel writes: "any omelet with the crucial characteristics would do as well," when I desire and find a perfect omelette. Next, Nagel says, "any person with the same flesh distribution" would not do equally well when it comes to sex. In sex, there is something more than sex, which I call love. This reveals the underlying moralism in Nagel's paper: I do not love omelets, except perhaps metaphorically, but I love my sex partner. This and only this explains the difference. ${ }^{15}$ Now, if we discuss sex qua sex one may well desire any potential partner who displays that same, lovely "flesh distribution." Sex is like that and that is why some people think of all sex as bad and dirty business. To make sex more palatable Nagel discusses ideal sex, which is nothing but sexually loaded type of love, although he shuns the term. He does not like to discuss love although he is unable to avoid it. In the modern urban secular world, sex and love are two separate things. The quest for mutual respect between the partners is another matter. Respect is a moral term and morality is always in demand. But love is too much to ask for.

\footnotetext{
$14 \quad$ Nagel, 1979, p. 42.

15 My wife suggests the following argument: A perfect omelet made by your loving wife is certainly preferable to a similar restaurant omelet.
} 


\section{Nagel: Ideally Good Sex, or in the Singles Bar}

What is the very best sex like then? Nagel argues, "[sex] involves a desire that one's partner be aroused by the recognition of one's desire that he or she be aroused." This tit-for-tat arousal sometimes happens but it is obviously too ambitious as a requirement, as Nagel recognizes, too. I wonder why he bothers to introduce the point. You and your partner may have mutually different sources and causes of arousal and yet your sex is certainly not perverted only because of that. However, if you two share "reciprocal interpersonal sexual awareness" you cannot go wrong. But what if I am aroused by the sight of your high heeled shoe and you are aroused by my arousal, independently of its source? How should one judge this exchange? It may be perverted, I suppose. Nagel may answer that the source of arousal must be perceived arousal, yet you must start somewhere. The question is, what is the relevant starting point of the cycle of arousals? What characteristics of the other person one should find initially attractive? What if I find only ugly persons sexy? What about their hair? If so, is it fetishism?

Ideal sex is always natural and normal, says Nagel. Is BDSM, as a test case, perverted by Nagel's standards? One thing is certain, however, BDSM can also be a mutually agreeable, freely chosen, and fully rewarding, mutually communicative sexual experience, as reported by the participants themselves. The top is aroused by the expressed pain of the bottom and the bottom is aroused by the top's aggression. Of course, Nagel may say that the sources of arousal here are not sexual in nature and not the same for both players, as they are in ideal vanilla sex: both partners are aroused by the sexual arousal of the partner, symmetrically. Is it important that the mutual sources of arousal are fully symmetrical? I do not understand why this should be so.

According to SP, a normal man goes to a bar and notices a nice looking normal woman who notices him. ${ }^{16}$ They spy on each other and both believe that one finds the other sexually attractive and displays the first signs of arousal. Anyway, all this leads them to a virtuous circle where he gets excited from her being excited and the same happens with her. ${ }^{17}$ Finally they both are sufficiently aroused to meet and talk, and then they to go to bed and satisfy their mutual lust: "For sexual arousal might begin with a person sensing that he is sensed being assailed by the perception of the other person's desire rather

16 Do singles bars still exist? (See https://punchdrink.com/articles/whatever-happened-tothe-singles-bar-tgi-fridays-maxwells-plum/). Today, sex traffic is on the net.

17 The paradoxes of this recursive approach are shown by R.D. Laing, Knots. New York: Vintage, 1970. 
than merely by the perception of the person. But there is a further step." In bed, we get pleasure from the pleasure of the other. The more the other enjoys the more I enjoy her enjoyment. In this way we both reach the maximal enjoyment. " $[\mathrm{H}] \mathrm{e}$ becomes conscious of his sexuality through his awareness of its effects on her and her awareness that this effect is due to him."18 Nagel is (too) strongly influenced by Jean-Paul Sartre here. Their concepts of flesh and sexuality do not come to an agreement. For Sartre, flesh meets flesh and becomes one, but for Nagel this cannot happen because one always must recognize the other, which entails a certain distance. Nagel's ideal couple never really fucks. Suppose I go to bed with my partner in order to see him or her react to my own sexuality. This may be important to me but it cannot be the point, if I am not a single minded narcissist or a pervert. Moreover, I do not understand what it means to become conscious of my own sexuality in this context. If one is a virgin, it may be fine but for an established husband or wife the situation is certainly different. As I see it, a sex act is for sexual satisfaction and orgasm. Yet I agree that sex is not only for sexual pleasure; sexual enjoyment is more that sexual pleasure as such.

I can of course see what Nagel means, namely, the moral ideal is a sexual encounter where the partners fully recognize each other as players of the same interactive game. However, many other ways to practice the best sex exist, also outside the singles bar, for instance, between well-established married couples. They need not spy on each other at a distance. Actually, anonymous sex between two strangers is seldom that good, or think of the glory hole. I wonder why Nagel makes his theory so dependent on mutual arousal and lust when he earlier hinted at the role of love. In a singles bar they as if serve omelets and other treats where the good distribution of flesh is all that counts. People there may avoid all references to love. I agree with Nagel that morally good sex requires that the partners at least recognize each other. But then, if that is the sole requirement, some perverts might achieve it as well. Nagel should somehow show that only natural and normal sex allows first for mutual recognition and then love.

The problems of the SP model of the best sex are then obvious. I mention two special problems. The first one is narcissism and, second, lack of sexual content. In the single's bar narrative the narcissistic aspect is overpowering. ${ }^{19}$ Next, the tit-for-tat model of mutual recognition may not be sexual. The only way out is to say that both parties are interested in sex and then they expect

18 Nagel, 1979, p. 46. Jon Nuttall in his Moral Questions: An Introduction to Ethics (Cambridge: Polity Press, 1993, p. 74) supports the same idea.

19 See J. Berman, Narcissism in Novel. New York: New York University Press, 1990, p. 21. 
to derive their forthcoming pleasure from it. Therefore, they interact with the other person in order to enjoy maximal mutual arousal. They interact to score, get laid, and receive their share of sexual pleasure. If they are moral individuals they fully respect the other's similar interests and they understand that without mutual recognition their own sexual enjoyment must be lacking. The bottom line is indeed sexual pleasure, not their mutual recognition or one's ability to make the other sexually aroused. The key point is that when the time is ripe for the sexual pleasure a moral person takes care of the partner; thus both may enjoy sex equally. Alas, the problem is that moral people, who alone are capable of the best sex, may not derive more sexual pleasure out of the sex act than bad people. They may feel better about their sexual exploits because they know they also gave pleasure, which is nice. They validly believe that they are not perverts, which is even nicer. A bad person may, alas, feel better when he thinks of her ability to non-consensually hurt and humiliate the partner - but this is not best sex according to Nagel.

\section{Nagel: the Genesis of Sexual Perversion}

Nagel's definition of sexual perversion in SP is something like this: A sexual inclination qualifies as a perversion if and only if it exists because of a particular psychological trauma in the person's earlier life. This presupposes a damaged person, or a pervert. Perhaps we can add: later in life the inclination becomes urgent and compulsive. For instance, this particular trauma has made the person unable to recognize another person's sexual arousal or to love him. Alternately, because of the trauma, the person's sexual focus is turned away from its vanilla objects; think of fetishism. Fetishism bypasses the recognition of the other as a person because the focus is on an object that is as such non-sexual. Let us now ask how Nagel argues for his definition.

Perverted sex is unnatural: "The concept of perversion implies that a normal sexual development has been turned aside by distorting influences" and "if humans will tend to develop some version of reciprocal interpersonal sexual awareness unless prevented, then the cases of blockade can be called unnatural or perverted." Here is then Nagel's famous theory of sexual perversion. Perversion means unnatural sex and unnatural means that a person's normal psycho-sexual development became distorted by some external influences or trauma so that the person, say, becomes unable to recognize the other. Notice that for Nagel people are perverted and abnormal when in the case of Freud acts and practices are perverted. For Nagel, a mentally healthy person cannot play perverted games; for Freud she can. But how can I know that this normal 
looking particular sex habit I am truly fond of, and which my partner seems to enjoy as well, is not the result of a trauma at some point of my psycho-sexual development? It is possible that my preference is a sexual perversion. Hence, I am unsure whether I am a pervert and in need of psychotherapy. Even when my sexual preferences are apparently normal they may be perverted. A trauma is not always remembered. Obviously, Nagel should first say that the trauma is the cause of dysfunctional sex, and then specify what is dysfunctional. But he only says that tit-for-tat arousal is functional, which is not enough.

Nagel writes, sex between normal persons can "achieve its goal on occasion" and thus provides "the conception of perversion with a foothold." What could this mean? As I see it, we sometimes have very good sex, as close to the ideal as humanely possible, which is impossible in the field of perversions (cf. the final chapter of this paper). Perversions may hedonistically be as good as you like, but still they somehow fail. Nagel's argument goes like this: The ideal and best sex is based on mutual recognition and arousal, perverts are unable to do so, hence their sex is always less than ideally good: "But if humans will tend to develop some version of reciprocal interpersonal sexual awareness unless prevented, then cases of blockage can be called unnatural or perverted." 20 This is what one can expect when the persons are perverted. Perverts may fare well but they can never reach perfection.

Hence, under Nagel's own criteria for instance consensual BDSM qualifies as a sexual perversion, to say nothing of non-consensual sadism, but his criteria seem ad hoc. He infers that BDSM players must be somehow damaged individuals. However, we have no idea of the natural, or free and spontaneous, development of human sexuality because early sex education is always socially controlled and even coercive. We have no idea what kind of sexuality Homo sapiens would develop were he and she fully unconstrained. Perhaps it would be wild, perhaps tame, and most likely promiscuous and bi-sexual. It may contain bestiality. The truth is that indigenous and aboriginal cultures employ wildly different sexual mores and practices. The Christian norms are not universal, nor is the Victorian heritage unquestionably natural, that much is clear. What is nearly universal is the multidimensional anxiety brought about by sex and sexuality in its non-intended audiences. It is true that all cultures control sex, even if Margaret Mead in Samoa did not realize it. ${ }^{21}$

$20 \quad$ Nagel, 1979, p. 49.

21 A particularly impressive account is in G.H. Herdt, The Guardians of the Flutes. New York: Columbia University Press, 1987, pp. 234ff.; and R.M. Berndt and C.H. Berndt, The First Australians. Canberra: Aboriginal Studies Press, 1999, Ch. vi. Think of the controversy over Margaret Mead's vision of perfectly free consensual sex in Samoa inspired by D. Freeman, Margaret Mead and Samoa: The Making and Unmaking of an Anthropological 
What Nagel means, as I see it, is that sexual perversions are trauma based obsessive-compulsive mental disorders. If the trauma causally determines one's sexual inclination, it will be repeated across all or most sexual contexts in one's life. Nagel's concept of sexual perversion is, thus, obsessive-compulsive; this is what he aims to explain - without mentioning or explicating this point. But, what if a pervert shows some flexibility in his abnormal preferences? Nagel is then in trouble. For instance, some S/M players freely switch between the roles of top and bottom, as Freud knows. Sometimes they like to watch. They are flexible even if sadism is a paradigm example of sexual perversions in SP. Compulsive rigidity is not a necessary condition of sexual perversity. Is it sufficient? Of course it is not. If I must kiss my wife in bed, I am not perverted only because of this.

Now, it is clear that sexual perversions are not necessarily rare or even uncommon. So we can forget the statistical meaning of abnormal. What about abnormal in the sense of violating a norm? This meaning fits the common idea of sexual perversion nicely, if we add like Freud that perversions are disgusting. As Nagel says, sex is always a regulated social affair. Sexual perversions are abnormal in the sense that they include sexual behaviour that violates important social norms. Perhaps sexual perversion designates sexual behaviour that breaks certain social norms of normal sexual behaviour. What if the norms is: Do not perform disgusting social acts, if the act is not vanilla sex? Such norms can be reasonable or unreasonable; popular or partisan; medical, religious, or legal; conventional or radical; traditional or novel; new or old. They may express a blind taboo, psychological disgust, elite opinion, medical community's scientific theory, or religious dogma - the opportunities are limitless. Moreover, the ideas of sexual perversion are malleable, as the homosexual narratives show. Another example is masturbation. ${ }^{22}$ When norms change certain forms of sex get liberated and when this happens more and more people find their variety tempting. People want to explore new possibilities because they feel free and unconstrained, which is fun. Incidentally, Nagel stumbles when he evaluates the status of homosexuality and sadism. Sadism is first a perversion "if anything is" and then sadism is a "really difficult case" to evaluate. About homosexuality, he recognizes that homosexual sex can be as good as

Myth. Cambridge, MA: Harvard University Press, 1983 . Mead was a sensation and Freeman a scandal because so many people wanted to believe in "good" sex at least in one place in the world; also see (https://www.psychologytoday.com/us/blog/the-imprinted -brain/201702/margaret-mead-and-the-great-samoan-nurture-hoax). Nagel's good sex is Mead's Samoan sex.

22 M. Foucault pays attention to the childhood masturbation panic in early 190os, see his Abnormal. Tr. G. Burgchell. London: Verso, 2003, Ch. 5 . 
heterosexual but then he says that it is possible that it is unnatural, depending on the genesis of the relevant desire. This is to say that of two similar sexual acts one may be perverted and one normal. The ambiguity is startling.

\section{Some Probable Reasons for the Success of SP}

Why did so many editors want to reprint SP? Why is it constantly mentioned in literature on sex? Why has SP been so enviably successful and still is? Who are the readers of the paper? Do people read it? How do they see it? What does it do to university students? These questions require an answer, because SP has its problems and shortcomings. It is a suggestive but carelessly argued paper. At the same time it obviously is an appealing piece of writing by a famous philosopher who has an excellent reputation all over the world, or at least wherever philosophy is studied and practiced. At the same time not many major philosophers have touched such issue as sex and sexuality, to say nothing of perversion, a taboo. The topic is more or less disgusting even in liberal countries, not in the sense that it could not be mentioned but in the sense that going too deep into case-studies, examples, and terminology is highly suspicious and also nauseating to many. SP avoids such pitfalls smoothly. It is written in a style that allows for no objection on the basis of obscenity. It is impossible to censor.

The main point, however, is that SP provides us with a reasoned conservative account of sexuality, its fringes, and those peculiar extensions that some of us still want to call perversions. SP is conservative, it detects an enemy, it says that the enemy is real, studies it, explains its genesis, and at least indirectly shows us the way out. This is done by telling what the ideal case of a sexual encounter is, or narrating the best sex. As we have seen, this is sex between undamaged strangers who fully recognize each other and derive their arousal and allegedly also their pleasure from the observation that they are able to excite each other sexually. This looks like a seductive scenario in the sense that both partners are fully equal, they interact in their mutual ways, and both partners have their full-fledged opportunity to sexual pleasure. Also, this scenario does not prescribe any special type of sexual activity, which may be anything you want and everything that is sexual and created via mutual recognition. This certainly allows for homosexuality but not for instance bestiality. However, Nagel certainly does not want to accept $\mathrm{S} / \mathrm{M}$, however consenting.

SP does not deserve the reputation it has. Perhaps the following facts explain its prolonged success: SP is written by a leading philosopher, SP deals 
with a rare topic in the English language philosophy, the topic is superbly interesting and emotionally challenging, the argument is conservative and as such minimally disturbing, the style is so abstract that no nasty sexual images emerge, the concept of ideal sex is pleasing to both sexes, the ideal sex is for middle-class, healthy, young, nice people who visit suitable social venues, frequent the dating sites on the net in search of sex and companionship, and most importantly, nothing much is said about sexual perversions. We find no descriptive accounts of perverse sex or even their names. SP paints a picture in which sexual perversion is just a name and a philosopher's little tame game that can be discussed at the dinner table. You may not want to eat all those strange dishes, but those who want to do so you know are traumatized. It is a consoling thought. They are damaged persons. The final consolation to the reader is that no one chooses their perversions freely. You, an honest, good, normal, middle-class person, are safe. Perversity always is the problem of the Other. You may want strange things in bed but it does not matter because you are not traumatized and you know it.

Finally, this is the main attraction of SP: it recommends sex as a moral affair that is intimately connected to recognition and love, which the good people enjoy more than the bad. Nagel preaches a moralistic model that also suits religious educators and responsible editors who want to look cool, calm, controlled, and enlightened. What has this to do with perversions and sexual perverts? Practically nothing, as I have tried to argue. If SP is not read critically its effect will be, as far as I can see, negative from the point of view of sexual liberty and mutual understanding between people of diverse sexual preferences and inclinations. I do not think we should label anyone psychologically injured and his or her sexual inclinations unnatural without thinking what we are doing. As I said, the basic rule is, also in the modern sexual jungle, condemn those who maltreat others and help those who suffer because of their own personal inclinations. ${ }^{23}$ That is all that should interest us. Let us not try to rationalize and promote personal prejudices about sex. ${ }^{24}$

23 Think of the poor narcissists, Berman, 1990, p. 18: "Behind narcissists' self-love lies selfhate, behind their grandiosity lies insecurity."

24 Even sexual therapists are not innocent of this fallacy. J. Virtanen in his sexology textbook Kliininen sexologia (Clinical Sexology) (Helsinki: wsoy, 2001) notices that some women find it easier to reach orgasm when their movements during sex are forcefully restricted. If this is a necessary condition for an orgasm, he says, the person is paraphilic (p. 115). This is a dubious claim. 


\section{Cases of Criticism}

Narcissism: A narcissist is psychologically incapable of the SP style best sex, this should be obvious, because her focus is always solely on herself. However, narcissism is not a sexual perversion; it is an ego or character problem. Now, according to SP, the best sex begins when you are aroused by your perception that the other is aroused because of you. Such a perception is a narcissist reaction, which is clear when one thinks of a non-veridical perception of the other. It is not necessary for you to be a proper narcissistic personality in order to find such a perception superbly appealing, although it helps. It may just appeal to your narcissist side. The same can be said about the other. Therefore, the first steps towards good sex are best taken by two proto-narcissists. Other persons are in trouble if they are unable to believe that they are so sexually attractive that such an attractive other could be serious in his/her admiration.

Now, think of what happens when you end up in bed with your new admirer. According to SP, you now get your enjoyment out of the situation where you please the other and bring about the maximal sexual pleasure to her, and she feels the same. In this case, you and the other behave like altruists for whom the pleasure of the other is the most important thing. What you aim at is the veridical perception that the other enjoys you in bed. The other thinks in the same way, so you two enjoy great and even ideal sex. Under these conditions the sexual encounter works well, as SP emphasizes. However, the psychological demands on you and the other obviously make an inconsistent set: a narcissist is now supposed to convert into an altruist. Your focus will change from yourself (he/she likes me) to your partner (I want to please him/her) so that the beginning calls for a narcissist and the end for an altruist. People can do that only if they are not true narcissists, otherwise they cannot. In this way the requirements are mutually incompatible. I do not think the quest for the presentation of such a double personality is a good idea. Think of an advice given to a young person: start as a narcissist and then switch over to altruism, and all will be fine, your partner will like you.

Sexual Pleasure: If I derive my sexual enjoyment in bed from pleasing the other, the situation looks abnormal, if not perverted. I please the other in bed when I should take care of my own sexual pleasure. I do not go to bed with the other to please her or see her react to my action for the simple reason that this is not a sexual motive. I go to bed in order the get my maximal sexual pleasure out of the situation, but given that I am a moral person, I do not want to satisfy myself regardless of the other's satisfaction, and certainly not at the expense of the other. In the first case I am a sexual predator and in the second an exploiter. My motive for having sex is sexual pleasure even if the morally laudable motive 
for having sex also recognises the other's similar interests. The bottom line is, therefore, that when I go to bed with the other I want sexual pleasure. Nagel writes, mysteriously, "if enjoyment is considered very important" in good sex; how could it not be? ${ }^{25}$

Is Nagel's SP style altruism in bed a perversion? It is not, according to how I understand perversions. However, according to the SP model of sexual perversion the matter is not so simple. Suppose we locate a psychological trauma in the life history of the sexual altruist that explains why he is not primarily seeking for his own sexual pleasure but that of the other and deriving his enjoyment from it. Because of his trauma he, as an altruist, is incapable of getting pleasure directly from sex. He always prefers the other's enjoyment to his own sexual pleasure. This just might qualify him as a sexual pervert. You must not go to bed to please the other. Your sexual behaviour is then unnatural. To exaggerate a little, you are the other's consenting sexual slave.

Explanation of Sexual Perversions: What is wrong with the explanation of the origin of sexual perversion in SP? Suppose a trauma T explains sexual perversion $\mathrm{P}$. This is possible but only if we know what $\mathrm{P}$ is, and SP says precious little about perversions, actually so little we do not know what they look like. However, if you want to use the SP model of their explanation you must first correlate $\mathrm{T}$ and $\mathrm{P}$. If the correlation is high enough you may proceed towards showing that $\mathrm{T}$ brings about $\mathrm{P}$. The trouble is that $\mathrm{SP}$ never says what $\mathrm{P}$ is like and thus we cannot establish any correlation between $\mathrm{T}$ and $\mathrm{P}$. We only learn that sadism is a perverse preference but not what the fully identifying description of sadism is. Therefore, SP can say only this much: given that you can identify the forms of sexual perversion you also can identify their causal history, which is explicable in terms of a mental trauma. Each trauma causally produces its corresponding perversion. Such an explanatory method is fine, but as I said you must first provide a descriptive account of perversions, which SP definitely fails to do. You cannot explain what you do not know.

Think of two persons A and B who share exactly the same sexual inclinations and the relevant behavioural patterns, say, they are sadists. A has suffered an identifiable and verifiable psychological trauma at some point of his early life, which explains his sexual perversion. B's history does not contain any similar traumatic events but yet he is a compulsive sadist, too. According to Nagel, we must say that B is not a sexual pervert, unlike A. In B's case her sadism is, say, a socially learned bad habit and thus we cannot call her a sexual pervert. It follows that often, or perhaps in most cases, we do not know whether a sadist is sexually perverted, because we cannot find a trauma in one's life history, or we 
cannot prove that his trauma is responsible for a sexual perversion this person happens to exhibit. The cause of the phenomenon is not part of the meaning of the phenomenon. If you break your leg by falling at home or on the football field, it is still just a broken leg. If you want to know what a broken leg is, it is not enough to talk about football games and say a broken leg is what you get when you play football. I should not call a doctor and say I have what one gets on the football field. She wants to know what I have.

Cultural relativism vs. scientific explanation: Nagel says that all cultures draw a distinction between good and bad sex, or normal or natural and twisted or perverted sex, although the patterns of good sex varies widely between the cultures. In our Christian, post-Victorian cultural environment we call tit-for-tat arousal based vanilla sex $(\mathrm{V})$ normal and natural. What a happy co-incidence it is that we can show scientifically that non-traumatized and undamaged persons tend to develop in this direction. Psychological trauma and developmental damage explain perverted character and desires, say, X.

Now, suppose that, in a temporally or ethnically distant culture K, people appreciate sexual behaviour X and condemn V. Scientifically speaking it follows that the members of $\mathrm{K}$ are somehow damaged individuals because only the relevant damage brings about such behaviour. It is true that distant cultures have social norms that deviate from our norms. This may show that perversion is a substantial concept but it also shows that the members of those cultures are psychological damaged perverts. The question is, how can we help them and make them understand the value of $\mathrm{V}$. These people are simply wrong when they disapprove and condemn $\mathrm{V}$ and prefer $\mathrm{X}$. I find this conclusion rather alarming. One should not mix cultural relativism and scientific objectivism.

In sum: I desire $\mathrm{X}$ and, therefore, I am a pervert in the scientific sense that I am a damaged individual. In culture $\mathrm{K}$, person $\mathrm{P}$ desires $\mathrm{X}$ and, therefore $\mathrm{P}$ is a pervert, too. $\mathrm{P}$ is a damaged individual. However, $\mathrm{P}$ has learned that $\mathrm{X}$ is good sex, but he is wrong. I have learned that $\mathrm{X}$ is not good sex and I am right. Yet, I cannot control my desires, which is typical of my illness.

Best sex: In the last two pages Nagel panics and attempts to save face. He as if condemned perversions in the main argument of the SP, but now he wants to take it all back. He said earlier that perverted sex, or sex by perverts, cannot be ideal sex. Now he writes, "It is not clear that unperverted sex is necessarily preferable to the perversions." This is to say, perverts can enjoy sex, or have as good sex as normal people, which is a strange idea. If a pervert is always and necessarily a damaged person, how can his/her sex be ideally good? If a pervert is a person who is damaged by the relevant trauma but their sex life is perfect, why speak of trauma at all? Can I really say, I am a damaged person but it does not matter? What is a damage that has no consequences? Of course, a pervert 
may like sadomasochistic games or homosexual encounters, unlike the normal person, but what does this matter if both fully enjoy beautiful sex?

In the end, SP self-destructs:

[E]ven someone who believed, for example, that homosexuality was a perversion could admit a distinction between better and worse homosexual sex, and might even allow that good homosexual sex could be better sex than not very good unperverted sex. If this is correct, it supports the position that, if judgements of perversion are viable at all, they represent only one aspect of the possible evaluation of sex, even qua sex. ${ }^{26}$

Even if the idea of perversion were valid, which is not certain, it does matter when we discuss sex. Why Nagel did write SP, becomes unclear. And he forgets that in SP he really discusses perverts, who are damaged individuals, not perversions, like Freud. Nagel does not evaluate sex or sexual behaviour; on the contrary, he wants to identify some damaged persons. Finally, the point is not what people believe about perverted sex because Nagel has given an objective and scientific criterion for identifying perverts among us. Homosexuality is perverted sex if homosexuals can be shown to be damaged by a developmental trauma. SP may be a popular read but its basic tenet is sinister. SP condemns certain persons by naming those perverts, and this without offering even one thought to healing. It is not enough to say that a damaged individual can have good sex, as long as it is bad sex. And it is bad sex because perversion is at the same time a substantial and pejorative term. The last two pages of SP indicate that Nagel himself comes close to realizing these problems, but all he does is to put up a smokescreen.

\section{Conclusion: Anxious Desires}

To conclude, let me quote two authors, first a self-confessed kinky person, a submissive woman, and the second, an author, scholar, and cultural critic. Kacie Cunningham defends her own lifestyle as follows; I have a hard time imagining a good counterargument.

So, are submissives born or made? The answer, as near as I can tell, is that submissives are, like all other people, created by the environment out of the raw material which is provided by nature. Some people would 
certainly like to claim that we are deviants, warped by childhood trauma, but I reject that. I say that we are what we are, regardless of how we got to be this way, and all that really matters is that we accept - and celebrate - ourselves. $^{27}$

However, a counterargument can be suggested. What could it be and how to evaluate it? Jon Nuttall writes:

$[T]$ here need be nothing wrong with sexual activity which is not normal. As I am using the term, to describe a sexual activity as perverted is to describe it as being other than what sex should be like and not simply other than what it is actually like. Perverted activities may or may not be normal [...]; they are, however, activities which I want to single out as being wrong [...]. The term "perverted," I suggest, is primarily a term to describe a person's moral character. ${ }^{28}$

This is to say $\mathrm{D} / \mathrm{S}$ is, or at least may be perverted and therefore morally wrong, hence Cunningham's argument may look suspicious. However, to say that perversions are what sex should not be like, and then say that the should here is the moral should, looks so strange that we need not take is seriously. What I am worried about is that Nuttall's book is a textbook. I do not think one can define perversion as sex that is morally wrong. The resulting list of perverted sexual desires will be too strange to be useful or even interesting. What Cunningham is saying is, leave me alone and let me live my life; certainly, the worst intrusion is to start asking whether she is an immoral character or not. Her own book provides ample proof to the effect that she is a good person.

Nuttall and Nagel reason in the same problematic way. What Nuttall should do is first to give an independent characterization of perverse sex, and then identify its typical features that make it morally wrong. But what he actually does is to define perversion as morally wrong and then classify all sexual desires and practices as perverse and not perverse on that basis. I wonder what this classification would be like. I must confess I have no idea. We saw above that Nagel reasons in this way, too. Think of the wide definition of rape today. Would Nuttall really call all those who are condemned of rape in, say, Sweden perverts?

Ronald Pearsall writes in his remarkable book on Victorian sex and sexuality:

27 K. Cunningham, Conquer Me. Eugene, OR: Greenery Press, 2012, p. 12.

28 Nuttall, 1993, p. 76. 
Perversion is a word that has no final meaning. What might be a perversion in one century can be normal in the following. It helps to consider it as a subjective term, denude it of its ethical overtones, and ask of a specific perversion: What physical harm it does? Then ask a subsequent question: What psychological harm does it do beyond satisfying the wishes of the protagonists?29

Pearsall is right: it does not make sense to say that a satisfied desire or wish alone may constitute that kind of psychological harm against which any ethically responsible person should fight - in a paternalistic fashion. Certainly one should not harm others in the course of sexual play and one may want to be careful with pleasures that harm the person herself. But if she ardently desires something that she knows harms herself in a way that she does not desire, she needs psychiatric help. This is the only such case I can imagine. Think for instance of cosmetic amputations. If one self-consistently wants them, the injury does not constitute harm to oneself. However, one may want the amputations when one does not accept the related injury, which entails harm to self. This leads to cognitive dissonance and in the long run to painful anxiety. Then it is mad to want cosmetic amputations, and perhaps, perhaps, perverted, too.

My conclusion is closely related to what Igor Primoratz says, namely, perversion is a "concept best discarded." ${ }^{30}$ If this is to say that the concept should be neither used nor mentioned, then we agree. Today we must not use or mention racist terms and the same applies to perversion. The idea of perversion is subjective, culturally and historically relative, pejorative, essentially contested, and inherently discriminatory. Originally it is a hate concept. Moreover one should not assume it covers unethical behaviour, criminal action, or mental illness.

29 R. Pearsall, The Worm in the Bud: The World of Victorian Sexuality. Harmondsworth: Pelican Books, 1971, p. 422; original italics.

30 I. Primoratz, Ethics and Sex. London: Routledge, 1999, p. 63. Incidentally, he says that M. de Sade discards the concept of perversion. This is not quite true because he discusses sexual crimes (Les Crimes de l'Amour), and according to him, it is this that makes sex worthwhile. Vanilla sex is worthless. 
Timo Airaksinen - 9789004410305

Downloaded from Brill.com04/26/2023 11:43:44AM via free access 